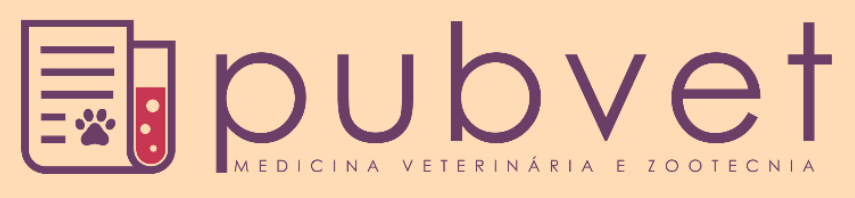

https://doi.org/10.31533/pubvet.v12n9a176.1-7

\title{
Estudo da comercialização de peixes ornamentais da família Loricariidae (Siluriformes) em Santarém/PA
}

\author{
Albino Luciano Portela de Sousa ${ }^{1^{*}}$, Luan Aércio Melo Maciel² ${ }^{\bullet}$, Luís Reginaldo \\ Ribeiro Rodrigues ${ }^{30}$
}

${ }^{I}$ Doutorando, Universidade Federal do Oeste do Pará, Programa de Pós-graduação em Sociedade, Natureza e Desenvolvimento, Santarém-PA, Brasil.

${ }^{2}$ Mestrando, Universidade Federal do Oeste do Pará, Programa de Pós-graduação em Biociências, Santarém-PA, Brasil.

${ }^{3}$ Professor Associado, Universidade Federal do Oeste do Pará, Instituto de Ciências da Educação, Santarém-PA, Brasil.

*Autor para correspondência E-mail: albinoportela@hotmail.com

\begin{abstract}
RESUMO. No Brasil, o comércio de peixes ornamentais de ambientes dulcícolas ainda depende essencialmente da atividade extrativista, principalmente na região amazônica. $\mathrm{O}$ estado do Pará se apresenta como um dos principais fornecedores de peixes ornamentais da família Loricariidae, sobretudo nas bacias do rio Xingu e Tapajós. No presente estudo analisamos a comercialização de peixes ornamentais da família Loricariidae oriundos do rio Tapajós e comercializados por empresas instaladas na cidade de Santarém. Os dados primários foram obtidos a partir das Guias de Transporte Animal (GTA) e Guias de Trânsito de Peixes com fins Ornamentais e de Aquariofilia (GTPON), perfazendo o período de janeiro/2013 a dezembro/2016. Foram comercializados um total de 136.705 exemplares que geraram a receita bruta de R \$365.013,80. As espécies Baryancistrus sp. (LDA33), Hypancistrus sp. (L260), Pseudacanthicus sp. (L273) e Peckoltia compta (L134) são as mais produtivas e economicamente rentáveis, sendo responsáveis por $59,6 \%$ da receita bruta gerada no período de janeiro/2013 a dezembro/2016. A produção de peixes ornamentais em Santarém/PA é oriunda da atividade extrativista da pesca artesanal e quase totalmente comercializada no mercado nacional. Os resultados obtidos são importantes para a compreensão da cadeia produtiva de peixes ornamentais da bacia do Tapajós, pois ainda existe pouco conhecimento publicado sobre a estrutura da pesca ornamental nesta região.
\end{abstract}

Palavras chave: acari, aquariofilia, cadeia produtiva, explotação, rio Tapajós

\section{Study of the Loricariidae family (Siluriformes) ornamental fish business at Santarém/PA}

ABSTRACT. In Brazil, the trade of ornamental fish from freshwater environments still relies on the essentially extractive activity, largely in the Amazon region. The Pará State is one of the major Loricariidae ornamental fish supplier, especially with the Xingu and Tapajós river basins. In the present study, we analyzed the Loricariidae ornamental fish trade from the Tapajós River, marketed by companies operating at Santarém city. The raw data were compiled from the official documents: Animal Transport Guides (GTA) and Fish Transit Guides for Ornamental and Aquarium Fisheries (GTPON), covering a period from January/2013 to December/2016. Were sold 136.705 fish units, which yielded the gross income of R\$365.013,80. The species Baryancistrus sp. (LDA33), Hypancistrus $s p$. (L260), Pseudacanthicus sp (L273) and Peckoltia compta (L134) were the most productive and economically profitable, accounting for $59.6 \%$ of the gross income in the period from January/2013 to December/2016. The ornamental fish production of Santarém/PA resulted from the extractive artisanal fishing and was almost fully sold into the Brazilian market. 
Since there is still scarce literature about the ornamental fishery in the Tapajós basin, these results are important for the understanding of the local productive chain.

Keywords: cascudo aquarium, productive chain, exploitation, Tapajós river

\title{
Estudio de la comercialización de peces ornamentales de la familia Loricariidae (Siluriformes) en Santarém/PA
}

\begin{abstract}
RESUMEM. En Brasil, el comercio de peces ornamentales de ambientes de agua dulce todavía depende esencialmente de la actividad extractivista, principalmente en la región amazónica. El estado de Pará se presenta como uno de los principales proveedores de peces ornamentales de la familia Loricariidae, sobre todo en las cuencas del río Xingu y Tapajós. En el presente estudio analizamos la comercialización de peces ornamentales de la familia Loricariidae oriundos del río Tapajós y comercializados por empresas instaladas en la ciudad de Santarém. Los datos primarios se obtuvieron a partir de las Guías de Transporte Animal (GTA) y las Guías de Tráfico de Peces con fines Ornamentales y de Acuariofilia (GTPON), que sumaron el período de enero/2013 a diciembre/2016. Se comercializaron un total de 136.705 ejemplares que generaron los ingresos brutos de $\mathrm{R} \$ 365.013,80$. Las especies Baryancistrus sp. (LDA33), Hypancistrus sp. (L260), Pseudacanthicus sp (L273) y Peckoltia compta (L134) son las más productivas y económicamente rentables, siendo responsables por el 59,6\% del ingreso bruto generado en el período de enero/2013 a diciembre/2016. La producción de peces ornamentales en Santarém/PA es oriunda de la actividad extractivista de la pesca artesanal y casi totalmente comercializada en el mercado nacional. Los resultados obtenidos son importantes para la comprensión de la cadena productiva de peces ornamentales de la cuenca del Tapajós, pues todavía hay poco conocimiento publicado sobre la estructura de la pesca ornamental en esta región.
\end{abstract}

Palabras clave: cascudo, acuariofilia, cadena productiva, explotación, río Tapajós

\section{Introdução}

A comercialização de peixes ornamentais no Brasil representa um importante setor econômico que, no início do século 21, movimentou um volume aproximado de 900 milhões de dólares (Cardoso \& Igarashi, 2009). Em 2005 este patamar atingiu 15 bilhões de dólares e estima-se que esta atividade econômica crescerá em média $14 \%$ ao ano (Moreau \& Coomes, 2007). Este mercado movimenta cerca de 400 milhões de exemplares por ano, sendo que cerca de $90 \%$ são peixes produzidos por criadores e $10 \%$ são provenientes da atividade pesqueira extrativista (Chapman, 2000).

O Brasil e a Colômbia são os principais exportadores de peixes ornamentais nativos da América do Sul. A atividade extrativista de peixes ornamentais do Brasil tem se concentrado principalmente na região amazônica, onde se destacam polos regionais como o município de Barcelos (AM) e Altamira (PA). Os estados do Amazonas e Pará são detentores de $88 \%$ das exportações derivadas de pesca extrativista (Araújo, 2016). No ano de 2015, as exportações brasileiras renderam mais de 9 milhões de dólares com a movimentação de cerca de 3 milhões de exemplares de peixes de água doce. Os principais destinos compradores de peixes ornamentais do Brasil são Alemanha, Estados Unidos, Hong Kong, Japão e Tailândia (Araújo et al., 2017).

Os peixes da família Loricariidae (Siluriformes) são caracterizados por possuírem o corpo recoberto por três ou mais séries de placas dérmicas, e por isso, recebem o nome popular de "cascudos" ou "acaris". Possuem a boca modificada em forma de disco ou ventosa, que geralmente são usadas para a fixação em substratos rochosos e troncos de madeira no leito dos rios (Silvano et al., 2001). Apresentam alto valor comercial e representam a terceira família mais comercializada no estado no Amazonas (Anjos et al., 2009; Araújo et al., 2017). No estado do Pará os principais polos fornecedores de cascudos ornamentais são as bacias do rio Xingu e Tapajós, pois as mesmas agregam uma vasta diversidade de espécies e apresentam peculiaridades, geográficas e hidrológicas, que favorecem a diversidade deste grupo (Araújo, 2016; Buckup \& Santos, 2010).

$\mathrm{O}$ rio Tapajós é formado pela confluência do rio Teles Pires com o rio Juruena, em Barra de São Manuel na fronteira entre Pará e Mato Grosso, e 
percorre uma extensão de aproximadamente 800 $\mathrm{km}$ até desaguar no Amazonas (ICMBIO, 2011). Informações sobre a comercialização de peixes ornamentais do rio Tapajós são escassas, e os poucos dados disponíveis não se encontram facilmente acessíveis tanto para a consulta acadêmica como para a disseminação do conhecimento junto a sociedade. No presente trabalho analisamos aspectos sócio econômicos da comercialização de peixes ornamentais (fam. Loricariidae) oriundos do rio Tapajós, tendo em vista a geração de conhecimentos aplicáveis a gestão de recursos naturais e na mitigação de impactos sobre os estoques deste valioso recurso pesqueiro.

\section{Material e Métodos}

A coleta dos dados foi obtida a partir da pesquisa documental utilizando como fonte as Guias de Transporte Animal (GTA) e Guias de Trânsito de Peixes com fins Ornamentais e de Aquariofilia (GTPON), ambos disponibilizados pela Agência de Defesa Agropecuária do Estado do Pará (ADEPARA) gerência Santarém-Pará.

Foram analisados os dados referentes aos períodos de janeiro de 2013 a dezembro de 2016. Os documentos incluem o nome das espécies, identificação comercial (código L), número de exemplares, preço unitário e total, local da compra (mercado nacional e internacional), nome do comprador (indivíduo/empresa) e procedência (extrativismo ou aquicultura).

A coleta de dados e análises se restringem aos peixes ornamentais da família Loricariidae cuja procedência situa-se na bacia do rio Tapajós. Com relação aos nomes científicos das espécies adotouse a nomenclatura citada na literatura corrente (Armbruster, 2004; Buckup \& Santos, 2010; Reis et al., 2006; Froese \& Pauly, 2018).

\section{Resultados}

Foram analisadas 343 GTA e 164 GTPON. No período de janeiro/2013 a dezembro/2016 foram comercializados a partir de Santarém/PA, 136.705 unidades de peixes ornamentais de 21 espécies da família Loricariidae (Figura 1, Tabela 1). No ano de 2013 foram comercializadas 11.389 unidades, seguidas por $26.645 \mathrm{em} \mathrm{2014,18.435} \mathrm{em} 2015$ e 80.236 em 2016. No período analisado observouse um crescimento de 7 vezes sobre o número inicial de unidades comercializadas. Neste período, as espécies mais exploradas foram Peckoltia compta (L134) com 24.614 unidades
(18\%), seguida de Hypancistrus sp. (L260) com 23.210 unidades (17\%). Algumas espécies não foram comercializadas no período de um ano (Hemiancistrus snethlageae, Scobiancistrus sp., Scobiancistrus pariolispos), dois anos (Ancistrus sp.) e três anos (Farlowella amazona).
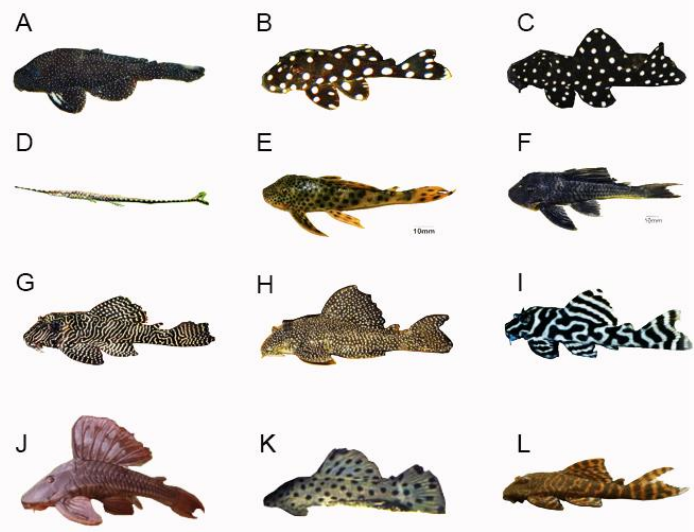

M

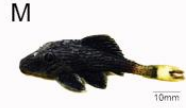

$\mathrm{N}$
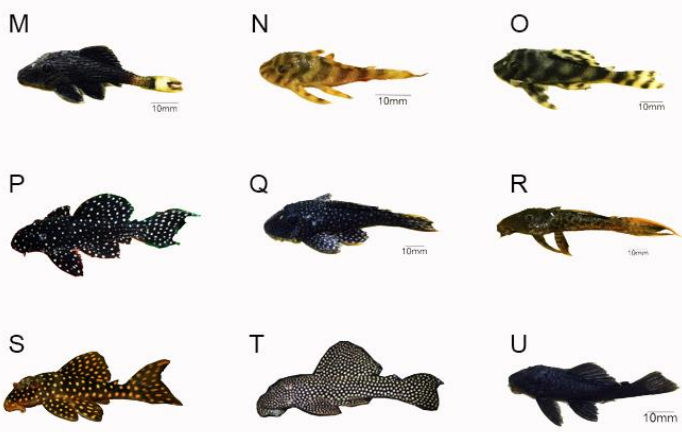

Figura 1. Ilustração da morfologia externa e padrão de colorido dos cascudos ornamentais comercializados em Santarém/PA. A. Ancistrus sp.; B. Baryancistrus sp. LDA 33; C. Baryancistrus sp. L142; D. Farlowella amazona; E. Hemiancistrus sabaji L075; F. Hemiancistrus snetlegeae L215; G. Hypancistrus sp. L260; H. Hypancistrus sp. L262; I. Hypancistrus sp. L333; J. Hypostomus soniae L137; K. Leporacanthicus joselimae L264; L. Panaque sp. L271; M. Panaque ambrusteri L27; N. Peckoltia compta L134; O. Peckoltia vittata L015; P. Pseudacanthicus sp. L097; Q. Pseudancistrus sp. 259; R. Pseudacanthicus sp. L273; S. Scobiancistrus sp. L048; T. Scobiancistrus pariolispos L133; U. Spectracanthicus murinus.

Os principais mercados consumidores nacionais foram as cidades de Belém, São Paulo e Manaus e os principais destinos internacionais foram os países de Hong Kong, China e Japão (Figura 2). No período de 2013 a 2016 a comercialização de peixes ornamentais loricarídeos, em Santarém/PA, gerou uma receita bruta de $\mathrm{R} \$ 365.013,80$. O ano de maior arrecadação foi 2016, cuja receita atingiu $\mathrm{R} \$$ $226.633,50$, o que coincide com o ano de maior volume de unidades comercializadas. Analisando o quantitativo financeiro por espécie, pode-se destacar a espécie Peckoltia compta (L134) que 
Tabela 1. Unidades de peixes ornamentais da família Loricariidae comercializadas em Santarém/PA e valor bruto de receita agregado por espécie, no período de 2013-2016.

\begin{tabular}{lcrrrrrr}
\hline Espécie & Identidade comercial & 2013 & 2014 & 2015 & 2016 & Total & Valor agregado (R\$) \\
\hline Ancistrus sp. & - & 30 & 0 & 161 & 0 & 191 & 346,00 \\
Baryancistrus sp. & LDA33 & 1350 & 3467 & 2796 & 9755 & 17368 & 39586,60 \\
Baryancistrus sp. & L142 & 195 & 295 & 25 & 8377 & 8892 & 35196,50 \\
Farlowella amazona & - & 70 & 0 & 0 & 0 & 70 & 70,00 \\
Hemiancistrus sabaji & L075 & 829 & 2122 & 1021 & 1531 & 5503 & 10145,30 \\
Hemiancistrus snethlageae & L215 & 620 & 597 & 0 & 2366 & 3583 & 5849,50 \\
Hypancistrus sp. & L260 & 1700 & 3219 & 3433 & 14858 & 23210 & 64591,70 \\
Hypancystrus sp. & L262 & 409 & 540 & 1000 & 4900 & 6849 & 14699,50 \\
Hypancistrus sp. & L333 & 470 & 398 & 1142 & 5675 & 7685 & 19955,25 \\
Hypostomus soniae & L137 & 453 & 1635 & 1001 & 2130 & 5219 & 8877,00 \\
Leporacanthicus joselimae & L264 & 732 & 993 & 903 & 5653 & 8281 & 19649,50 \\
Panaque sp. & L271 & 70 & 560 & 30 & 1356 & 2016 & 3764,50 \\
Panaque ambrusteri & L027 & 585 & 988 & 1285 & 664 & 3522 & 8326,00 \\
Peckoltia compta & L134 & 1180 & 9943 & 2905 & 10586 & 24614 & 70116,00 \\
Peckoltia vittata & L015 & 980 & 557 & 630 & 1860 & 4027 & 8202,35 \\
Pseudacanthicus sp. & L097 & 89 & 239 & 249 & 1335 & 1912 & 5357,00 \\
Pseudacanthicus sp. & L273 & 1075 & 760 & 1105 & 7326 & 10266 & 43318,00 \\
Pseudancistrus sp. & L259 & 95 & 14 & 129 & 662 & 900 & 1412,00 \\
Scobiancistrus sp. & L048 & 140 & 79 & 0 & 155 & 374 & 2888,60 \\
Scobiancistrus pariolispos & L133 & 0 & 83 & 263 & 462 & 808 & 1035,00 \\
Spectracanthicus murinus & - & 317 & 156 & 357 & 585 & 1415 & 1564,50 \\
\hline Total & & 11389 & 26645 & 18435 & 80236 & 136705 & 365013,80 \\
\hline & & & & & &
\end{tabular}

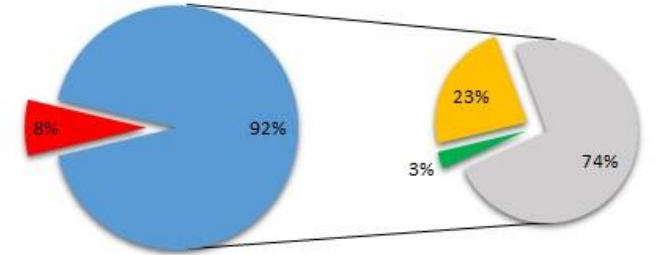

Mercado Internacional Belém - São Paulo Manaus

Figura 2. Principais destinos dos peixes ornamentais da família Loricariidae explorados no rio Tapajós.

movimentou o maior valor financeiro cerca de $\mathrm{R} \$ 70.116,00$ seguida pelo Hypancistrus sp. (L260) com um montante de $\mathrm{R} \$ 64.591,70$ e pelo Pseudacanthicus sp. (L273) com a quantia de R\$ 43.381,00 (Tabela 1). Em geral, todas as espécies apresentaram uma valorização do preço/unidade ao longo do período estudado, entretanto, algumas espécies apresentaram enormes variações de preço/unidade, como por exemplo, Scobinancistrus sp. (L048) cujo valor mínimo passou de $\mathrm{R} \$ 1,00$ para $\mathrm{R} \$ 31,00$, assim como Hypancistrus sp. (L333) comercializada inicialmente por $\mathrm{R} \$$ 0,80/unidade em 2013 para
R\$ 27,50/unidade em 2016, Pseudacanthicus sp. (L273), por sua vez teve preço $\mathrm{R} \$ 0,05 /$ unidade elevado para $\mathrm{R} \$ 25,00 /$ unidade (Tabela 2).

\section{Discussão}

As espécies ornamentais Peckoltia compta (L134, Teoro ou Tigre), Hypancistrus sp. (L260, Acari Pão) e Baryancistrus sp. (LDA33, Bola de Neve) foram os peixes loricarídeos mais explorados comercialmente em Santarém/PA no período de 2013-2016, representando 47,8\% das unidades (indivíduos) comercializadas e correspondendo a $47,8 \%$ da receita bruta deste empreendimento.

Pela análise documental registramos a ocorrência de dois táxons denominados Baryancistrus sp., comercialmente identificados como LDA33 e L142; porém, de acordo com a Instrução Normativa Interministerial No. 001/2012 os dois códigos são atribuídos a mesma espécie. Portanto, a exploração de Baryancistrus sp. no mercado de peixes ornamentais em Santarém/PA contribuiu com a comercialização de 26.260 unidades $(19,2 \%)$, o que a coloca no ranking de segunda espécie mais explotada, 
superada apenas por Peckoltia compta. Por outro lado, Baryancistrus sp. gerou a receita bruta de $\mathrm{R} \$$ $74.783,31$, o que a torna a espécie de maior valor comercial no mercado de peixes ornamentais de Santarém/PA, no período 2013-2016.

Em geral houve aumento da produtividade na maioria das espécies, com um crescimento acentuado no último ano do período. Das 20 espécies relacionadas apenas três mostraram declínio do número de unidades comercializadas:
Farlowella amazona, Panaque ambrusteri (L027) e Pseudacanthicus sp. (L273). Das quatro espécies mais comercializadas $P$. compta foi a que apresentou a maior variação do número de unidades no período analisado (Figura 3). A captura de cascudos ornamentais não é regular durante o ano, isto porque a abundância de cada espécie é influenciada, não somente pela disponibilidade de áreas de coleta, mas também, por questões peculiares da região amazônica, tais como, a sua área geográfica e seus ciclos hidrológicos (ㅅaújo, 2016).

Tabela 2. Preço mínimo e máximo de comercialização de peixes ornamentais da família Loricariidae.

\begin{tabular}{lccc}
\hline Espécie & Identidade comercial & Preço mínimo & Preço máximo \\
\hline Ancistrus sp. & & 1,00 & 3,00 \\
Baryancistrus sp. & LDA33 & 1,00 & 15,00 \\
Baryancistrus sp. & L142 & 1,00 & 3,00 \\
Farlowella amazona & - & 1,00 & 1,00 \\
Hemiancistrus sabaji & L075 & 1,00 & 11,85 \\
Hemiancistrus snethlageae & L215 & 0,50 & 3,00 \\
Hypancistrus sp. & L260 & 0,80 & 20,00 \\
Hypancystrus sp. & L262 & 1,00 & 5,00 \\
Hypancistrus sp. & L333 & 0,80 & 27,45 \\
Hypostomus soniae & L137 & 0,15 & 4,00 \\
Leporacanthicus joselimae & L264 & 1,00 & 18,00 \\
Panaque sp. & L271 & 1,00 & 3.00 \\
Panaque ambrusteri & L27 & 1,00 & 18,00 \\
Peckoltia compta & L134 & 1,00 & 6,00 \\
Peckoltia vittata & L015 & 1,00 & 15,42 \\
Pseudacanthicus sp. & L097 & 1,00 & 10,00 \\
Pseudacanthicus sp. & L273 & 0,05 & 25,00 \\
Pseudancistrus sp. & L259 & 1,00 & 3,00 \\
Scobiancistrus sp. & L048 & 1,00 & 31,00 \\
Scobiancistrus pariolispos & L133 & 1,00 & 3,50 \\
Spectracanthicus murinus & - & 0,50 & 3,00 \\
\hline
\end{tabular}

No Brasil, o comércio de peixes ornamentais de água doce ainda depende essencialmente da atividade extrativista, que constitui um importante setor econômico em várias cidades da região amazônica, tais como, Barcelos (AM) localizada na bacia do rio Negro e Altamira (PA) na bacia do rio Xingu (Anjos et al., 2009). Na bacia do Tapajós, a cidade de Itaituba é referida como importante polo exportador de peixes ornamentais (Camargo et al., 2005).

No presente estudo, evidencia-se o crescimento deste setor na cidade de Santarém, situada na confluência dos rios Tapajós e baixo Amazonas.

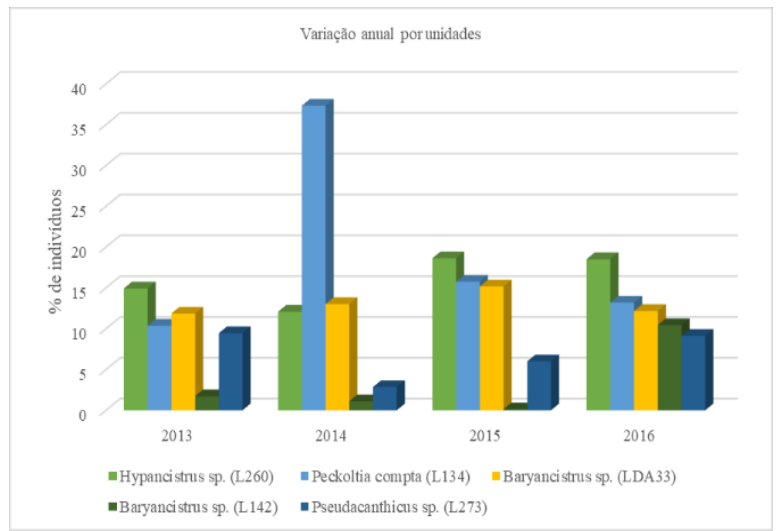

Figura 3. Variação anual do número de indivíduos comercializados das principais espécies ornamentais da família Loricariidae explotadas em Santarém/PA no período 2013-2016. 
A atividade extrativista de peixes ornamentais é considerada potencialmente prejudicial à preservação da biodiversidade, visto que algumas das espécies exploradas são desconhecidas do ponto de vista taxonômico e ecológico, ou seja, existe uma carência de estudos básicos sobre a biologia de várias espécies comercializadas (Araújo, 2016). O principal marco regulatório desta atividade econômica é a Instrução Normativa Interministerial 001/2012. Dentre as espécies comercializadas em Santarém, apenas Ancistrus sp. não está citada para uso comercial pela referida IN.

O preço/unidade de peixe ornamental apresenta variação ao longo do ano devido a inúmeras variáveis, tais como, regime de cheias e vazante dos rios, quantidade disponível do pescado para aquele ano (demanda $\mathrm{x}$ oferta) e condições de logística da cadeia produtiva e de comercialização. De acordo com Prang (2007) a cadeia de comercialização deste recurso natural exibe uma grande diferença de preço em cada nível e essa variação contribui com a oscilação de renda nas comunidades provedoras e pela ausência de sustentabilidade da atividade, visto que provoca a necessidade de grandes quantidades capturadas para obtenção de um nível mínimo de renda (Araújo et al., 2017).

A maior parte dos produtos da pesca ornamental brasileira é destinada ao mercado consumidor internacional, sendo que os principais destinos estão localizados na Europa, América do Norte e na Ásia (Prang, 2007). Os estados do Pará e Amazonas concentram $88 \%$ das exportações de peixes ornamentais com captura extrativista do país (Araújo et al., 2017).

Apesar do declínio gradual do Brasil nas exportações ao nível internacional de exemplares de peixes ornamentais de água doce no período de 2006 a 2015 (Figura 4), com perda de aproximadamente $89 \%$ do volume total exportado (Ferreira, 2016), este padrão de declínio não se ajusta aos dados observados na comercialização de peixes ornamentais loricarídeos da bacia do Tapajós, o que se caracteriza por um vertiginoso crescimento tanto na produção quanto nos valores de receita associados.

Uma das explicações possíveis para o aumento da exploração de peixes ornamentais do rio Tapajós pode estar ligada ao declínio nos últimos 10 anos do comércio e da pesca de peixes ornamentais na cidade de Barcelos no estado do Amazonas (Ferreira, 2016) e, por isso, a necessidade de buscar novas áreas que ainda são pouco exploradas e que apresentam uma vasta diversidade de espécies, como é o caso bacia do rio Tapajós, na qual pode se configurar em nível nacional como uma importante área de exploração extrativista de peixe ornamental de água doce.

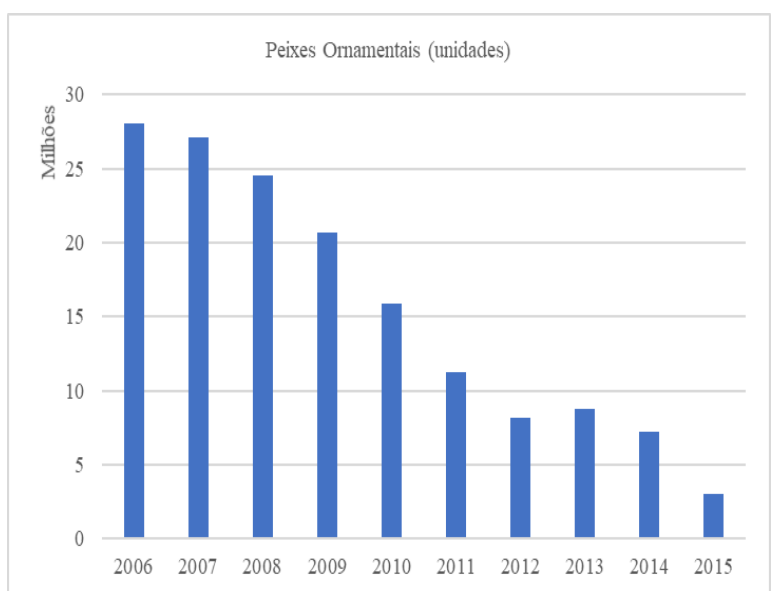

Figura 4. Volume de exportação de peixes ornamentais brasileiros no período de 2006-2015. Dados modificados de Ferreira (2016).

Os dados relativos à comercialização de peixes ornamentais na bacia do rio Tapajós ainda são incipientes comparados a de outras bacias da região amazônica, como a bacia do rio Xingu. A cidade de Santarém possui a melhor infraestrutura de portos e aeroportos na região do Tapajós e, portanto, poderá alavancar o crescimento da cadeia produtiva local. Vislumbra-se a necessidade de investimentos neste setor econômico, de modo a promover a modernização das instalações e logística do transporte, a organização dos atores que compõe a cadeia produtiva, o fortalecimento das políticas voltadas para a exploração e conservação deste recurso natural, aproximação do meio acadêmico com vistas a geração de conhecimentos científicos demandados para o melhor uso e agregação de valor aos produtos comercializados (peixes ornamentais).

\section{Conclusões}

A bacia do rio Tapajós representa importante polo produtivo de peixes ornamentais da família Loricariidae com oferta diversificada de pelo menos 21 espécies legalmente autorizadas para a explotação. As espécies Baryancistrus sp. (LDA33), Hypancistrus sp. (L260), Pseudacanthicus sp. (L273) e Peckoltia compta (L134) são as mais produtivas e economicamente rentáveis, sendo responsáveis por $59.6 \%$ da receita bruta gerada no período de janeiro/2013 a 
dezembro/2016. A produção de peixes ornamentais em Santarém/PA é quase totalmente comercializada no mercado nacional.

\section{Agradecimentos}

Ao ICMBIO e ADEPARA por conceder acesso as bases documentais. As empresas AMAZON FISH e TAPAJÓS AQUÁRIOS pelo compartilhamentos de dados comerciais. À CAPES pela bolsa pela bolsa de mestrado concedidas para LAMM. Ao Programa CAPESPROAMAZÔNIA, AUXPE 3318/2013O pelo financiamento do projeto "Estudos Citogenéticos e Citogenômicos da Biodiversidade da Amazônia com implementação de avanços técnicos" que permitiu realizar o estudo.

\section{Referências Bibliográficas}

Anjos, H. D. B., Siqueira, J. A., Amorim, R. M. S., \& Anjos, C. R. 2009. Exportação de peixes ornamentais do estado do Amazonas, Bacia Amazônica, Brasil. Boletim da Sociedade Brasileira de Ictiologia, 35, 259-274.

Araújo, J. G. 2016. Economia e pesca de espécies ornamentais do Rio Xingu, Pará, Brasil. Universidade Federal do Pará, Belém, Pará, Brasil.

Araújo, J. G., Santos, M. A. S., Rebello, F. K., \& Isaac, V. J. 2017. Cadeia comercial de peixes ornamentais do Rio Xingu, Pará, Brasil. Boletim do Instituto de Pesca, 43(2), 297-307.

Armbruster, J. W. 2004. Phylogenetic relationships of the suckermouth armoured catfishes (Loricariidae) with emphasis on the Hypostominae and the Ancistrinae. Zoological Journal of the Linnean Society, 141(1), 1-80.

Buckup, P. A., \& Santos, G. M. 2010. Ictiofauna da Ecorregião Xingu-Tapajós: fatos e perspectivas. Boletim da Sociedade Brasileira de Ictiologia, 98, 3-9.

Camargo, M., Giarrizzo, T., \& Carvalho Júnior, J. 2005. Levantamento ecológico rápido da fauna ictica de tributários do Médio-baixo Tapajós e Curuá. Boletim do Museu Paraense Emílio Goeldi, 1(2), 213-231.

Cardoso, R. S., \& Igarashi, M. A. 2009. Aspectos do agronegócio da produção de peixes ornamentais no Brasil e no mundo. PUBVET, $3(15), 1-22$.

Chapman, F. A. 2000. Ornamental fish culture, freshwater. Encyclopedia of Aquaculture, 3, 602-610.

Ferreira, V. A. M. 2016. Avaliação da pesca e comércio de peixes ornamentais no município de Barcelos, Amazonas, Brasil. Master of Science, Universidade de Aveiro, Aveiro, Portugal.

Froese, R.; D. \& Pauly. 2018. Editors. FishBase.

ICMBIO - Instituto Chico Mendes de Conservação da Biodiversidade. Levantamento da Ictiofauna, Caracterização Genética e da Fauna Parasitária da bacia hidrográfica do rio Tapajós. 2011.

Moreau, M.-A., \& Coomes, O. T. (2007). Aquarium fish exploitation in western Amazonia: conservation issues in Peru. Environmental Conservation, 34(1), 12-22.

Prang, G. 2007. An industry analysis of the freshwater ornamental fishery with particular reference to the supply of Brazilian freshwater ornamentals to the UK market. Scientific Magazine UAKARI, 3(1), 7-52.

Reis, R. E., Pereira, E. H. L., \& Armbruster, J. W. 2006. Delturinae, a new loricariid catfish subfamily (Teleostei, Siluriformes), with revisions of Delturus and Hemipsilichthys. Zoological Journal of the Linnean Society, 147(2), 277-299.

Silvano, R. A. M., Oyakawa, O. T., Amaral, L. B. D., \& Begossi, A. 2001. Peixes do Alto Rio Juruá (Amazonas, Brasil) (Vol. 1). São Paulo, Brasil: EDUSP.

Recebido: 11 Agosto, 2018.

Aprovado: 27 Agosto, 2018

Publicado: 18 Setembro, 2018

Licenciamento: Este artigo é publicado na modalidade Acesso Aberto sob a licença Creative Commons Atribuição 4.0 (CC-BY 4.0), a qual permite uso irrestrito, distribuição, reprodução em qualquer meio, desde que o autor e a fonte sejam devidamente creditado. 